NKU-2021-SF1

\title{
Thermodynamics and Heat Engines of Black Holes with Born-Infeld-type Electrodynamics
}

\author{
Leonardo Balart 1 \\ Departamento de Ciencias Físicas, \\ Facultad de Ingeniería y Ciencias \\ Universidad de La Frontera, Casilla 54-D \\ Temuco, Chile. \\ Sharmanthie Fernandd 2 \\ Department of Physics, Geology \& Engineering Technology \\ Northern Kentucky University \\ Highland Heights, Kentucky 41099, U.S.A.
}

\begin{abstract}
In this paper we have studied electrically charged black holes in a new model of nonlinear electrodynamics introduced by Kruglov in Ref. [1]. There are two parameters for the theory and the black hole could have up to two horizons. Thermodynamics is studied in the extended phase space where the pressure is proportional to the cosmological constant. First law and the Smarr formula are derived. There are phase transitions similar to the Van der Waals liquid-gas phase transitions. Black hole is also studied as a heat engine and have discussed how the parameters in the nonlinear electrodynamics theory effect the efficiency of the heat engine.
\end{abstract}

Key words: Static black hole; non-linear electrodynamics; heat engine; anti-de Sitter space; efficiency

\section{Introduction}

The Born-Infeld theory [2] is a model of non-linear electrodynamics which was proposed to avoid singularities of the Maxwell model. This theory is characterized by a non-linear parameter $b$ which represents the value of the electromagnetic field strength at the origin of a point source. In the limit $b \rightarrow \infty$ the Maxwell electrodynamics is recovered. This theory has received attention from different contexts [3 11]. In recent times alternative theories of nonlinear electrodynamics have been presented:

\footnotetext{
${ }^{1}$ leonardo.balart@ufrontera.cl

${ }^{2}$ fernando@nku.edu
} 
some of them are also characterized by a nonlinear parameter 12 18]. Other models have emerged in the context of regular black holes $[1924]$. However, as shown in Ref. 25] they cannot include a nonlinearity parameter that allows to obtain the Reissner-Nordström solution in the limit where it tends to infinity, as in the case of the nonlinearity parameter $b$ of the Born-Infeld electrodynamics, or as in the black hole solution with nonlinear electrodynamics given in Ref. [26], where the ReissnerNordström case is recovered when the nonlinearity parameter tends to zero.

Recently a black hole coupled to a Born-Infeld type electrodynamic model has been proposed in Ref. 27] that, in addition to the parameter $\beta$ which is related to the maximal electromagnetic field strength, depends on another extra parameter, denoted by $\sigma$, and where the Born-Infeld black hole corresponds to one of the values of $\sigma$. This new family of solutions has two characteristics that make it interesting in order to deepen its study. On the one hand, depending on the values of the extra parameter, it is possible to have solutions of an electric field that can be amplified (where therefore the regularity at the origin is lost) or screened with respect to the electric field of the Reissner-Nordström solution. This is something that deserves attention, for a better understanding about vacuum polarization in the context of black holes itself [28, 29], as well as its relationship with the deviation of the electric field with respect to the Coulomb field. On the other hand, it is also worth analyzing the model from the point of view of the energy conditions that the respective energymomentum tensor satisfies [30]. In particular, fulfilment of the dominant energy condition depends on the values that the parameter $\sigma$ takes. In respect of the latter, the relationship that the screening or the amplification of the electric field may have with the energy conditions that the corresponding energy-momentum tensor fulfills can be studied. More details on this will be presented in a later work.

One of the goals in this paper is to study the electrically charged black hole in this new electrodynamics in the extended phase space with $P=-\frac{\Lambda}{8 \pi}$. In the extended phase space, first law of black hole thermodynamics has an extra tern corresponding to $V d P$. Here $M=U+P V$ is the enthalpy and not the internal energy $U$. Many black holes have demonstrated phase transitions and critical behavior between large and small black holes in this context. Such phase transitions are similar to van der Waals liquid-gas phase transitions. There are large number of work related to this topic: we would only give few of such work as references as $[28,31,42]$. There is a review by Kubiznak et.al. [43] which gives more references and a comprehensive review on the topic.

It should be mentioned that several works have considered AdS black holes as heat engines [44 54]. Here the central idea is that if the extended phase space is considered one can extract mechanical work from a AdS black hole when one consider it as a heat machine. To compute the efficiency of the heat engine one must define a thermodynamic cycle in phase space. In this paper we will present a Born-Infeld-type black hole given by Kruglov [1] as a heat engine choosing one of the cycles proposed by Johnson in Ref. [44. This cycle is composed of two isobaric and two isochoric 
paths.

The paper is organized as follows: in section 2, the black hole considered is presented. In section 3 thermodynamics of the black holes are given and in section 4 $\mathrm{P}-\mathrm{V}$ criticality is discussed. In section 5 the black holes are studied in the context of heat engines and finally in section 6 the conclusion is given.

\section{Black holes with Born-Infeld-type electrodynam- ics}

In general the action of the 3+1-dimensional Einstein theory coupled with a nonlinear electrodynamics model is given by

$$
S=\int d^{4} x \sqrt{-g}\left[\frac{(R-2 \Lambda)}{16 \pi G}+\mathcal{L}(\mathcal{F})\right]
$$

Here, $g$ is the determinant of the metric tensor and $\Lambda=-\frac{3}{l^{2}}$ is the cosmological constant and $\mathcal{L}(\mathcal{F})$ is the the Lagrangian depending on the invariant $\mathcal{F}=F_{\mu \nu} F^{\mu \nu}$. The variation of the action with respect to the gravitational field yields the Einstein equations

$$
\begin{gathered}
G_{\mu \nu}+\Lambda g_{\mu \nu}=8 \pi T_{\mu \nu} \\
T_{\mu \nu}=g_{\mu \nu} \mathcal{L}(\mathcal{F})-F_{\mu \rho} F_{\nu}{ }^{\rho} \mathcal{L}, \mathcal{F} .
\end{gathered}
$$

And the variation with respect to the electromagnetic field gives

$$
\nabla_{\mu}\left(F^{\mu \nu} \mathcal{L}, \mathcal{F}\right)=0
$$

where $\mathcal{L}, \mathcal{F}$ is the derivative of $\mathcal{L}(\mathcal{F})$ with respect to $\mathcal{F}$.

In the present study we will restrict ourselves to the nonlinear electrodynamic model described by the Lagrangian density

$$
\mathcal{L}(\mathcal{F})=\beta^{2}\left[1-\left(1+\frac{\mathcal{F}}{\beta^{2} \sigma}\right)^{\sigma}\right] .
$$

This Lagrangian density can be obtained from the electrodynamic model introduced by Kruglov in Ref. [1] and whose Lagrangian density is given as

$$
\mathcal{L}(\mathcal{F})=\frac{1}{\beta}\left[1-\left(1+\frac{\beta \mathcal{F}}{\sigma}-\frac{\beta \gamma \mathcal{G}^{2}}{2 \sigma}\right)^{\sigma}\right]
$$

where $\mathcal{F}=F_{\mu \nu} F^{\mu \nu}, \mathcal{G}=\frac{1}{4} F_{\mu \nu} \tilde{F}^{\mu \nu}$ and $\beta, \gamma, \sigma$ are parameters of the theory. In particular by choosing $\gamma=0$ and doing the replacement $\beta \rightarrow 1 / \beta^{2}$ in Eq. (6), let us obtain Eq. (5). 


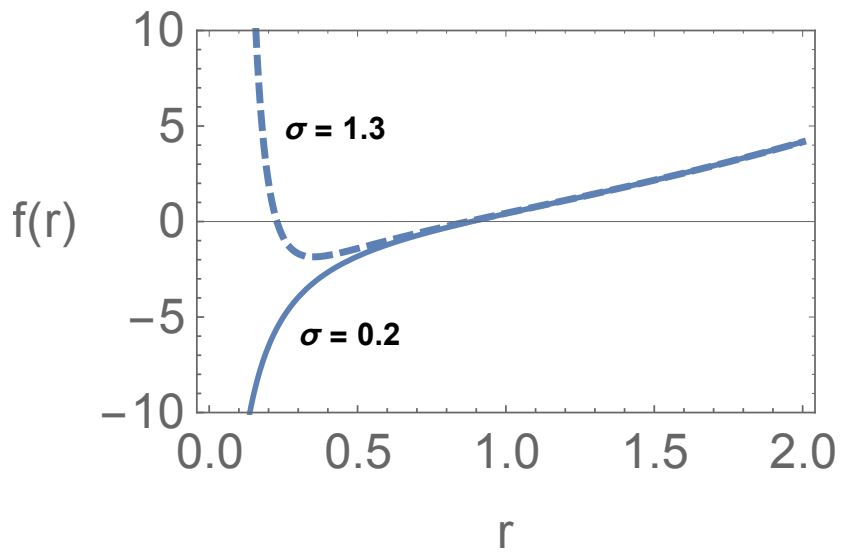

Figure 1: The figure shows $f(r)$ vs $r$. Here $q=0.434, M=0.878$, and $\beta=0.425$. The plots correspond to two values of $\sigma$ as given in the figure.

When $\beta=\gamma$ and $\sigma=\frac{1}{2}$ in Eq. (6), the model becomes the Born-Infeld electrodynamics. Hence the above new model is named Born-Infeld-type electrodynamics. A two parameter model was considered in Ref. [14] which is close to the above mentioned model. In Ref. [27], Kruglov investigated a magnetic black hole arising from the above model coupled to general relativity. In Refs. [14,55 57], the possible constraints for the parameters of this model (and also of other nonlinear electrodynamic models) are analyzed.

Static spherically symmetric black holes for the above theory is given by the metric $d s^{2}=-f(r) d t^{2}+f^{-1}(r) d r^{2}+r^{2}\left(d \theta^{2}+\sin ^{2} \theta d \phi^{2}\right)$. This ansatz in the Einstein equations (2) and using the Lagrangian density (5) allows us to obtain the metric function

$$
f(r)=1-\frac{2 M}{r}+\frac{r^{2}}{l^{2}}-\frac{2 \beta^{2} r^{2}}{3}\left[{ }_{2} F_{1}\left(-\frac{3}{4},-\sigma ; \frac{1}{4} ;-\frac{q^{2}}{2 \sigma \beta^{2} r^{4}}\right)-1\right]
$$

where $M$ represents the ADM mass, $q$ the electric charge and ${ }_{2} F_{1}(a, b ; c ; z)$ is the Gauss hypergeometric function. In Fig. 1, the function $f(r)$ is plotted for two values of $\sigma$. For small $\sigma$, the black hole has one horizon and behaves similar to the Schwarzschild AdS black hole. For large $\sigma$, the black hole could have two horizons and behave as the Reissner-Nordström-AdS black hole.

The electromagnetic tensor takes the form $F_{\mu \nu}=E(r)\left(\delta_{\mu}^{t} \delta_{\nu}^{r}-\delta_{\nu}^{t} \delta_{\mu}^{r}\right)$, where $E(r)$ is the electric field, that is, $\mathcal{F}=-2 E^{2}(r)$, which together with Eq. (4) allows us to obtain the corresponding electric field for the Born-Infeld-type electrodynamics

$$
E(r)=\frac{2 q r^{2} \beta^{2} \sigma}{q^{2}+2 \sigma \beta^{2} r^{4}}\left(1+\frac{q^{2}}{2 \sigma \beta^{2} r^{4}}\right)^{\sigma}
$$




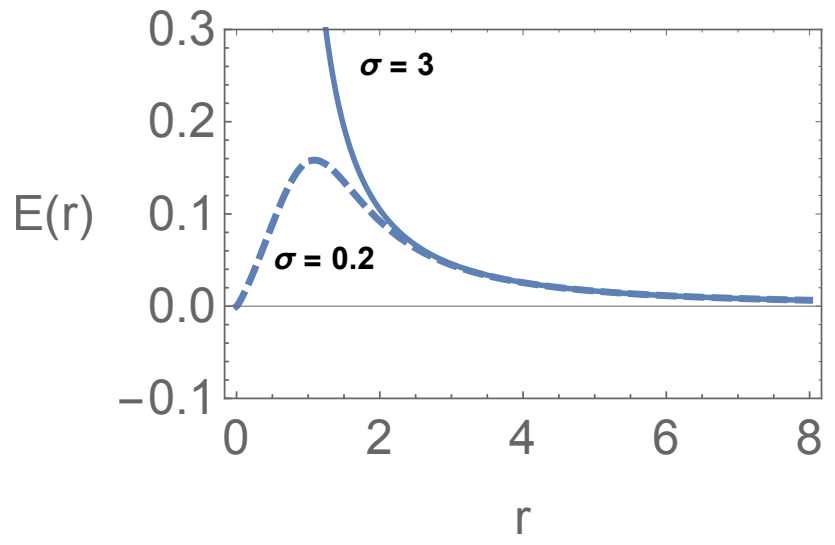

Figure 2: The figure shows $E(r)$ vs $r$. Here $q=0.411$ and $\beta=0.425$. The plots corresponds to two values of $\sigma$ as given in the figure.

The electric field asymptotically behaves as the one for the Reissner-Nordström-AdS black hole as,

$$
E(r)=\frac{q}{r^{2}}-\frac{q^{3}(1-\sigma)}{2 \sigma \beta^{2} r^{6}}+O\left(1 / r^{10}\right)
$$

In Fig. 2, the electric field is plotted for two values of $\sigma$. For small $\sigma$, the electric field is finite at $r=0$ similar to Born-Infeld electrodynamics. For large $\sigma$, the electric field is infinite at $r=0$ similar to Coulomb electric field.

\section{Thermodynamics of the black hole with Born-Infeld- type electrodynamics}

In this section, we will derive thermodynamic quantities, first law and the Smarr formula for the Born-Infeld-type black hole. We will be studying thermodynamics in the extended phase space where the thermodynamic pressure is given by $P=$ $-\Lambda /(8 \pi)=3 /\left(8 \pi l^{2}\right)$. The conjugate quantity to $P$ is $V=\frac{4 \pi r_{+}^{3}}{3}$. The Hawking temperature is given by (after replacing $l$ in terms of $P$ ),

$$
T=\frac{1}{4 \pi}\left|\frac{d f(r)}{d r}\right|_{r_{+}}=\frac{1}{4 \pi r_{+}}\left[1+8 \pi P r_{+}^{2}+2 r_{+}^{2} \beta^{2}\left(1-\left(1+\frac{q^{2}}{2 r_{+}^{4} \beta^{2} \sigma}\right)^{\sigma}\right)\right]
$$

where $r_{+}$is the black hole horizon. The entropy of the black hole, $S=\pi r_{+}^{2}$ is conjugate to the temperature.

The black hole horizon $r_{+}$satisfies $f\left(r_{+}\right)=0$. Then from Eq. (7), we can arrive at a formula for $r_{+}$as a function of the parameters $l, q \beta, \sigma$ and the ADM mass $M$. 
And from here we obtain the following expression for $M$

$$
M=\frac{r_{+}}{6}\left[-2 r_{+}^{2} \beta_{2}^{2} F_{1}\left(-\frac{3}{4},-\sigma ; \frac{1}{4} ;-\frac{q^{2}}{2 \sigma \beta^{2} r_{+}^{4}}\right)+8 \pi P r_{+}^{2}+2 r_{+}^{2} \beta^{2}+3\right]
$$

The electric potential $\Phi$ on the outer horizon, which is conjugate to the charge $q$ is computed as,

$$
\Phi\left(r_{+}\right)=\int_{r_{+}}^{\infty} E(r) d r=\frac{\beta^{2} r_{+}^{3}}{2 q}\left[\left(1+\frac{q^{2}}{2 \sigma \beta^{2} r_{+}^{4}}\right)^{\sigma}-{ }_{2} F_{1}\left(-\frac{3}{4},-\sigma ; \frac{1}{4} ;-\frac{q^{2}}{2 \sigma \beta^{2} r_{+}^{4}}\right)\right]
$$

We have defined a new quantity $B$ as the conjugate quantity to $\beta$. To write the first law, we will treat $M$ as the enthalpy given by $M=U+P V$. Here, $U$ is the internal energy of the black hole.

Rewriting the mass $M$ as

$$
M(S, P, q, \beta)=\frac{\sqrt{S}}{6 \sqrt{\pi}}\left[-\frac{2 S \beta^{2}}{\pi}{ }_{2} F_{1}\left(-\frac{3}{4},-\sigma ; \frac{1}{4} ;-\frac{\pi^{2} q^{2}}{2 \sigma \beta^{2} S^{2}}\right)+8 P S+\frac{2 S \beta^{2}}{\pi}+3\right],
$$

the differentiation allows us to obtain

$$
d M=\left(\frac{\partial M}{\partial S}\right)_{q, P, \beta} d S+\left(\frac{\partial M}{\partial P}\right)_{S, q, \beta} d P+\left(\frac{\partial M}{\partial q}\right)_{S, P, \beta} d q+\left(\frac{\partial M}{\partial \beta}\right)_{S, q, P} d \beta
$$

with

$$
\left(\frac{\partial M}{\partial S}\right)_{q, P, \beta}=T \quad\left(\frac{\partial M}{\partial q}\right)_{S, P, \beta}=\Phi \quad\left(\frac{\partial M}{\partial P}\right)_{S, q, \beta}=V
$$

which are compatible with the expressions given in Eqs. (10) and 12 and the volume of the black hole. We have also defined a new quantity $B$ as the conjugate quantity to $\beta$ following Refs. 28, 58]. Thus, we can compute the variable $B$ as,

$$
B=\left(\frac{\partial M}{\partial \beta}\right)_{S, q, P}=\frac{\beta r_{+}^{3}}{6}\left[4-{ }_{2} F_{1}\left(-\frac{3}{4},-\sigma ; \frac{1}{4} ;-\frac{q^{2}}{2 \sigma \beta^{2} r_{+}^{4}}\right)-3\left(1+\frac{q^{2}}{2 \sigma \beta^{2} r_{+}^{4}}\right)^{\sigma}\right] .
$$

Consequently, these quantities satisfy the first law of thermodynamics

$$
d M=T d S+V d P+\Phi d q+B d \beta
$$

From Eq. (13), we could obtain the Smarr formula following the scaling argument given in Ref. [59]. Carrying out the dimensional analysis, we note that the quantities obey the following scaling relations $S \propto l^{2}, P \propto l^{-2}, q \propto l^{1}$ and $\beta \propto l^{-1}$, where $l=$ [lenght], therefore the mass is a homogeneous function of degree 1 . Thus, the Euler theorem implies

$$
M=(2)\left(\frac{\partial M}{\partial S}\right) S+(-2)\left(\frac{\partial M}{\partial P}\right) P+(1)\left(\frac{\partial M}{\partial q}\right) q+(-1)\left(\frac{\partial M}{\partial \beta}\right) \beta
$$


So one can write the Smarr formula [60] as

$$
M=2 T S-2 V P+\Phi q-B \beta
$$

Recently, some results on thermodynamic relations have been obtained for black holes with nonlinear electrodynamics. For example, in Ref. [61] the author considers the solution given in Ref. [19], and uses a different thermodynamic approach than we consider, where the thermodynamic volume of the black hole is not the usual $4 \pi r_{+}^{3} / 3$, thus achieving to obtain a first law without adding new quantities and according to this the Smarr formula turns out to be $M=2 T S-2 V P+\Phi q$. In Ref. 62] a new black hole solution with nonlinear electrodynamics is presented, which depends on a nonlinearity parameter such that if it is zero then the Maxwell case recovers. Here, as in Refs. 26, 28] and our thermodynamic approach, a new term appears in the Smarr formula that is related to the quantity conjugated to the nonlinearity parameter. For its part, in Ref. [63], the first law and the Smarr formula are modified when a black hole with non-linear electrodynamics is considered, due to a new thermodynamic quantity, the surface tension. Also in the context of black hole solutions with nonlinear electrodynamics, in Refs. [64, 65], the first law is modified with a quantity that multiplies $d M$. In Ref. [66] it is considered that the entropy for regular black holes consists of two terms, one of them the usual and the other corresponds to a deviation, and when the black hole solution is obtained by coupling a nonlinear electrodynamic model, then it is also considered a corrected term for the potential electric. As future work one could take those thermodynamic approaches that are different from the one we are considering in the present work and make a detailed analysis for the case of black holes with Born-Infeld-type electrodynamics.

\section{$4 \quad$ P-V criticality of the black hole}

In this section, we will study the behaviour of pressure. The equation of state for a Born-Infeld-type black can be described from Eq. (10) as

$$
P=\frac{2 r^{2} \beta^{2}\left[\left(1+\frac{q^{2}}{2 \sigma \beta^{2} r_{+}^{4}}\right)^{\sigma}-1\right]+4 \pi r_{+} T-1}{8 \pi r_{+}^{2}}
$$

For certain values of parameters in the theory, there are critical values for $\mathrm{P}$ vs $r_{+}$ graph as shown in Fig. 3. In order to calculate the critical values and to study law of corresponding states, we first identify specific volume $v=2 r_{+}$. Then replacing $r_{+}=\frac{v}{2}$ in Eq. (20), the equation of state can be rewritten as follows:

$$
P=\frac{-1+2 \pi T v+\frac{v^{2} \beta^{2}}{2}\left((1+A)^{\sigma}-1\right)}{2 \pi v^{2}}
$$




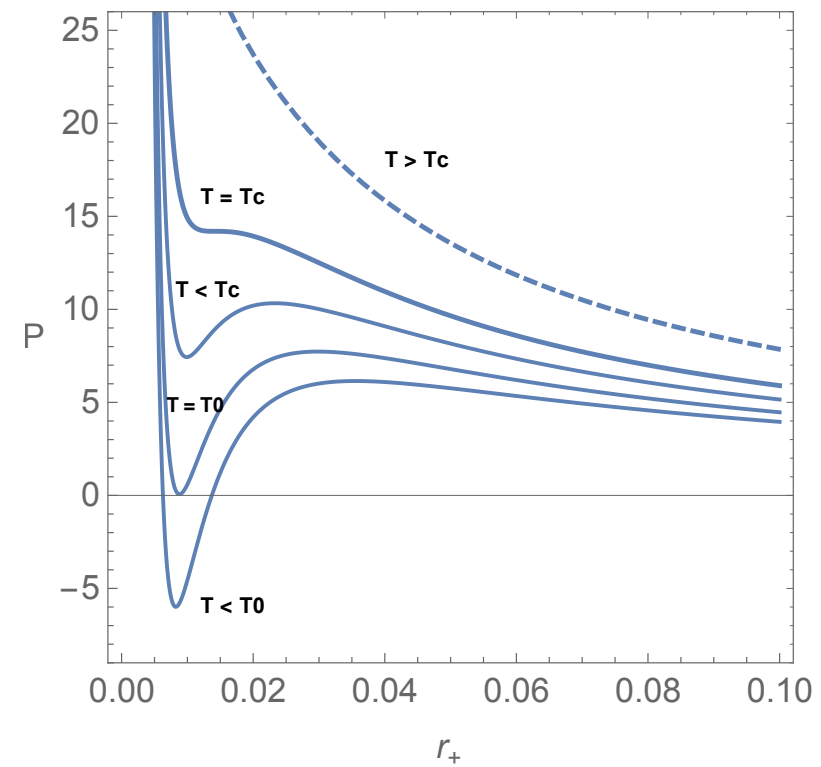

Figure 3: The figure shows $P$ vs $r_{+}$for varying temperature. Here $\sigma=0.539, q=0.41$, and $\beta=0.536$

where $A=\frac{8 q^{2}}{v^{4} \beta^{2} \sigma}$. Since $v \propto r_{+}$, the graph in Fig. 3 will be similar in shape to a graph for $P$ vs $v$. Critical value $t_{c}$ occurs when the $P$ vs $v$ graph has an inflection point. At the critical point,

$$
\frac{\partial P}{\partial v}=\frac{\partial^{2} P}{\partial v^{2}}=0
$$

Solving the two equations obtained due to the conditions in Eq. (22), critical temperature $T_{c}$ and critical pressure $p_{c}$ are given as follows:

$$
\begin{gathered}
T_{c}=\frac{v_{c}^{4} \beta^{2} \sigma+q^{2}\left(8-8 v_{c}^{2} \beta^{2}\left(1+A_{c}\right)^{\sigma} \sigma\right)}{\pi v_{c}\left(8 q^{2}+v_{c}^{4} \beta^{2} \sigma\right)} \\
P_{c}=\frac{-2+4 \pi T_{c} v_{c}+v_{c}^{2} \beta^{2}\left(-1+\left(1+A_{c}\right)^{\sigma}\right)}{4 \pi v_{c}^{2}}
\end{gathered}
$$

Here $A_{c}=\frac{8 q^{2}}{v_{c}^{4} \beta^{2} \sigma}$. It is not possible to solve the equations arising due to Eq. 22 completely for $v_{c}$ due to the nature of the equations. However, one could use Mathematica to solve for $P_{c}, T_{c}$ and $v_{c}$ for numerical values for $\beta$ and $\sigma$. The number $\frac{P_{c} v_{c}}{T_{c}}$ is an interesting number to calculate. In the Van der Waals fluids, $\frac{P_{c} v_{c}}{T_{c}}=\frac{3}{8}$ is a universal number for all fluids. It was shown that the Reissner-Nordström-AdS black hole undergo criticality in Ref. [31 and the value for $\frac{P_{c} v_{c}}{T_{c}}$ is $\frac{3}{8}$. In the current work, we have computed $\frac{P_{c} v_{c}}{T_{c}}$ numerically for various values of $\sigma$ and have plotted in Fig. 4. It is observed that the ratio $\frac{P_{c} v_{c}}{T_{c}}$ increases with $\sigma$. For $\sigma<1, \frac{P_{c} v_{c}}{T_{c}}<\frac{3}{8}$ and for $\sigma>1$, $\frac{P_{c} v_{c}}{T_{c}}>\frac{3}{8}$. 


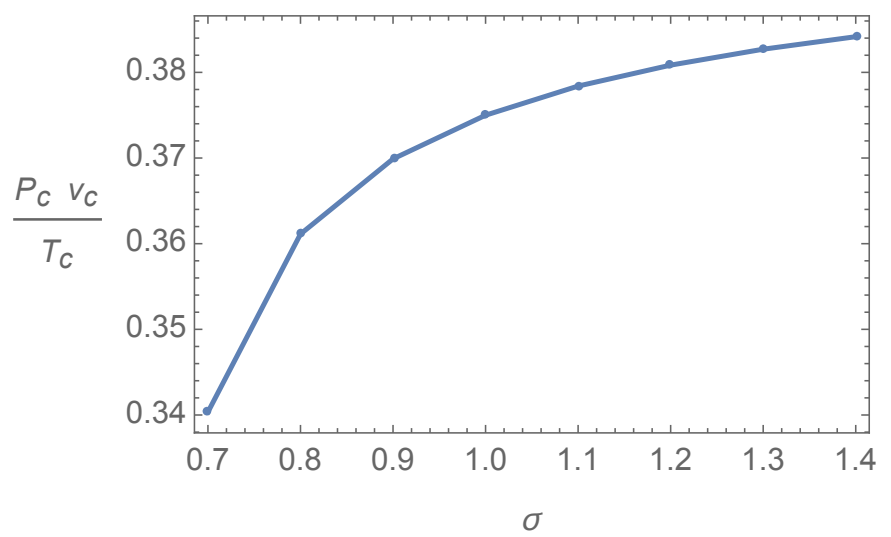

Figure 4: The figure shows $\frac{P_{c} v_{c}}{T_{c}}$ vs $\sigma$. Here $q=0.41$, and $\beta=0.536$

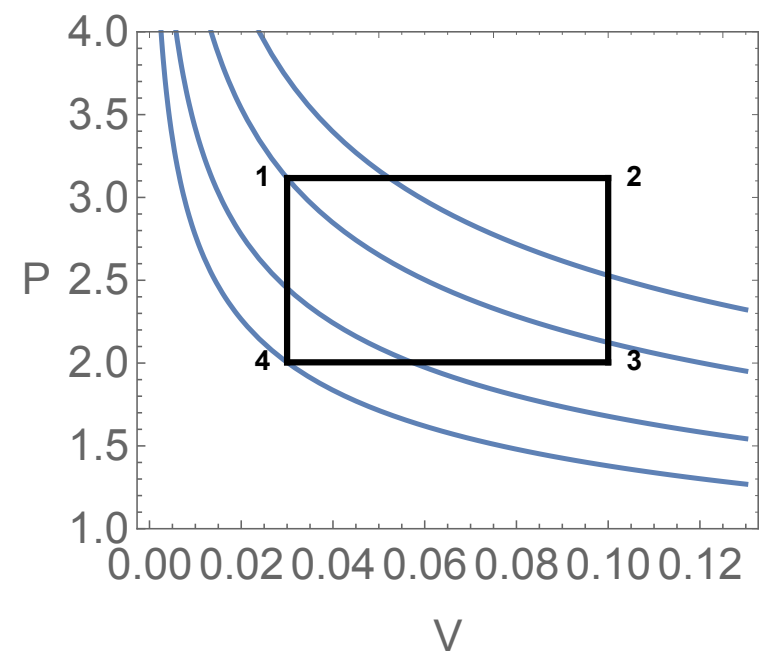

Figure 5: The figure shows $\mathrm{P}$ vs $\mathrm{V}$ for isothermals for varying temperatures. Here $q=1, \sigma=0.35$, and $\beta=0.412$ 


\section{Born-Infeld-type black hole as heat engine}

Black holes could be used as heat engines as demonstrated in several works mentioned in the introduction $44-54$. Since an equation of state is clearly defined with a pressure term and a volume, the $P d v$ term could be utilized to produce work. In order to create a heat engine, one need a thermodynamic cycle: we will use a thermodynamical cycle consisting of two isobaric and two isochoric paths as given in Fig. 5. Heat will be supplied along the path $1 \rightarrow 2$ and heat is out from the path $4 \rightarrow 3$. According to Ref. [47] the efficiency for the heat engine for such an engine is given by $\eta=1-\frac{M_{3}-M_{4}}{M_{2}-M_{1}}$. If we use Eq. (11), the efficiency is obtained as

$$
\eta=\frac{8\left(P_{1}-P_{3}\right) \sqrt{S_{3}} \sqrt{S_{4}}\left(S_{3}+\sqrt{S_{4}} \sqrt{S_{3}}+S_{4}\right)}{\sqrt{S_{3}} \sqrt{S_{4}}\left(8 P_{1}\left(S_{3}+\sqrt{S_{4}} \sqrt{S_{3}}+S_{4}\right)+3\right)-3 \pi q^{2}+F\left(S_{3}, S_{4}\right)} .
$$

where

$$
\begin{aligned}
& F\left(S_{3}, S_{4}\right)=\frac{1}{\pi\left(\sqrt{S_{3}}-\sqrt{S_{4}}\right)}\left\{\sqrt { S _ { 3 } } \left[2 S_{4}^{2} \beta^{2}\left({ }_{2} F_{1}\left(-\frac{3}{4},-\sigma ; \frac{1}{4} ;-\frac{\pi^{2} q^{2}}{2 S_{4}^{2} \beta^{2} \sigma}\right)-1\right)\right.\right. \\
& \left.\left.+3 \pi^{2} q^{2}\right]-\sqrt{S_{4}}\left[2 S_{3}^{2} \beta^{2}\left({ }_{2} F_{1}\left(-\frac{3}{4},-\sigma ; \frac{1}{4} ;-\frac{\pi^{2} q^{2}}{2 S_{3}^{2} \beta^{2} \sigma}\right)-1\right)+3 \pi^{2} q^{2}\right]\right\} .
\end{aligned}
$$

Note that when $\beta \rightarrow \infty, F\left(S_{3}, S_{4}\right)=0$. Hence, $\eta \rightarrow \eta_{0}$ where,

$$
\eta_{0}=\frac{8\left(P_{1}-P_{3}\right) \sqrt{S_{3}} \sqrt{S_{4}}\left(S_{3}+\sqrt{S_{4}} \sqrt{S_{3}}+S_{4}\right)}{\sqrt{S_{3}} \sqrt{S_{4}}\left(8 P_{1}\left(S_{3}+\sqrt{S_{4}} \sqrt{S_{3}}+S_{4}\right)+3\right)-3 \pi q^{2}} .
$$

Here $\eta_{0}$ is the efficiency of the Reissner-Nordström-AdS black hole solution. Also notice that,

$F\left(S_{3}, S_{4}\right)>0$ and $\eta<\eta_{0}$, if $\sigma<1\left(\sigma=\frac{1}{2}\right.$ Born-Infeld case) $F\left(S_{3}, S_{4}\right)<0$ and $\eta>\eta_{0}$, if $\sigma>1$

We have plotted $\eta$ vs $\sigma$ in Fig. 6. It is clear that when $\sigma$ increases, $\eta$ increases. We have also plotted $\eta$ vs $\beta$ in Fig. 7. When $\beta$ increases, $\eta$ also increases. Hence for fixed $\beta$, large $\sigma$ yields greater $\eta$.

\section{Conclusions}

In this paper, we have studied black hole solutions in a new non-linear electrodynamics model introduced by Kruglov [1]. There are basically two types of black holes for this new model: for small $\sigma$ the black hole behaves as the Schwarzschild-AdS black hole, and, for large $\sigma$, the black hole behaves as the Reissner-Nordström-AdS black hole. When $\sigma=1 / 2$, the black hole simplifies to the Born-Infeld black hole. 


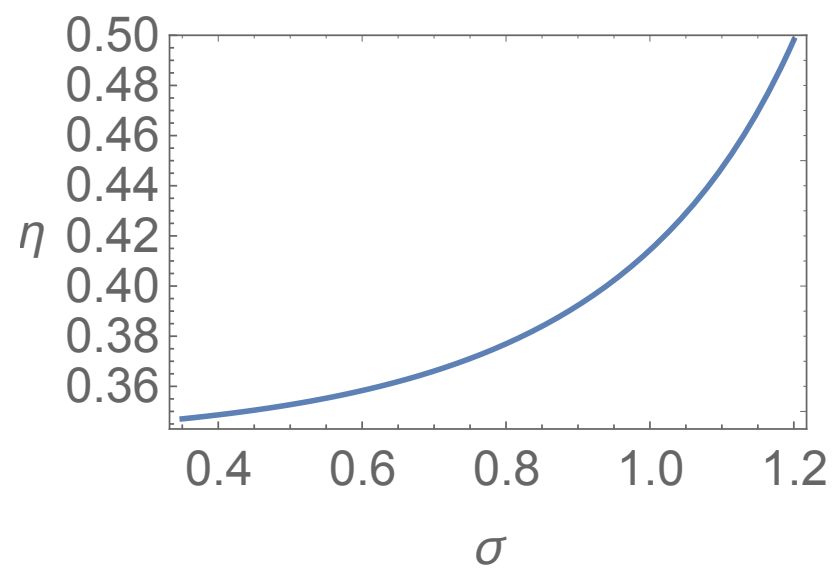

Figure 6: The figure shows $\eta$ vs $\sigma$. Here $q=1, \beta=0.412, P_{1}=3.117, P_{3}=2.004, S_{3}=$ 1.199 , and $S_{4}=0.537$

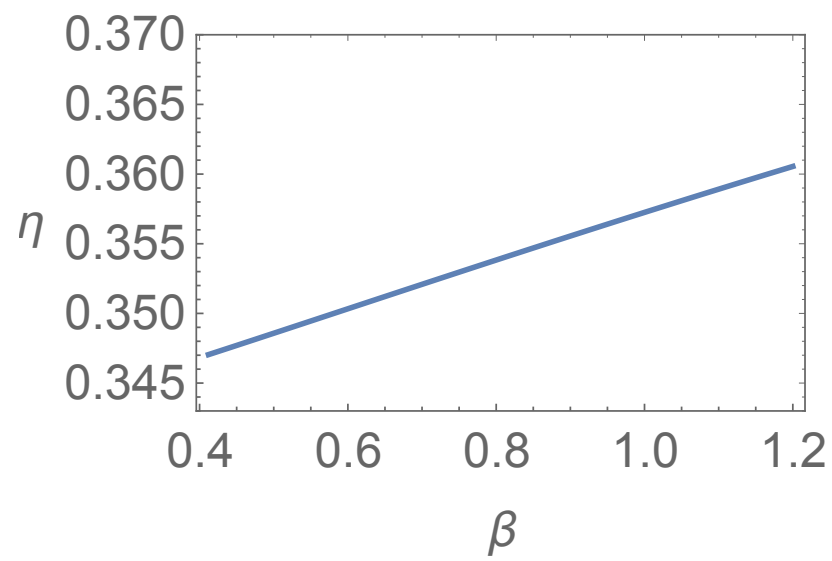

Figure 7: The figure shows $\eta$ vs $\beta$. Here $q=1, \sigma=0.35, P_{1}=3.117, P_{3}=2.004, S_{3}=$ 1.199, and $S_{4}=0.537$ 
Thermodynamics of the black hole is studied in the extended phase space where $P=-\frac{\Lambda}{8 \pi}$. First law and the Smarr formula are derived: here, there is a new thermodynamical quantity introduced as a conjugate quantity to $\beta$. We have shown that the black hole demonstrate phase transitions similar to van der Waals liquid-gas phase transitions. These black holes are between small and large black holes. It is observed that the ratio $\frac{P_{c} v_{C}}{T_{c}}$ is smaller than the universal values for liquids, $\frac{3}{8}$, when $\sigma<1$. For large $\sigma>1$ the ratio is large than $\frac{3}{8}$.

In Ref. [28], it was indicated that the quantity $B$ in the case of Born-Infeld black holes has units of electric polarization per unit volume. Such quantity is called BornInfeld polarization. In this same reference it is shown that $B$ is always positive and also noted that its physical meaning is not yet fully understood. For the model we have considered, it can be shown that $B\left(r_{+}\right) \geq 0$ if $\sigma \leq 1$ and $B\left(r_{+}\right)<0$ if $\sigma>1$. It would be interesting to ask about the interpretation of $B$ when its value is negative. We leave this aspect of the theory for future work.

We have studied these black holes as heat engines. The thermodynamical cycle considered for the heat engine is a rectangle in $P, V$ space with two isochoric and two isobaric processes. The efficiency of the heat engine, when considered the black hole as a heat engine, is studied. At this point, as a global conclusion, we can point out the relationship between the efficiency and the electric field, in particular with the values it takes with respect to the Coulomb field if we consider the same electric charge in both cases. Thus, via a numerical analysis, we can observe that by varying the parameter $\sigma$, which determines the regularity of the electrodynamic model, and keeping $\beta$ fixed (for the same electric charge), the efficiency increases together with the amplification of the electric field with respect to the Coulomb electric field. We also observe that in the region of parameter values corresponding to non-regular electric field solutions, the efficiency takes its highest values, for this family of Born-Infeld type solutions; unlike what happens in the region of regularity of the electric field, where the efficiency decreases as the electric field is increasingly screened with respect to the Coulomb electric field (the Born-Infeld case belongs to this region). In this region of regularity, we can also notice that as the value of the intercept of the curve of the electric field graph decreases, at the same time the efficiency decreases.

Additionally, let us indicate that within each of the two regions: of regularity or non-regularity, which are determined by the parameter $\sigma$ and in no case of $\beta$. The electric field can increase or decrease by varying the parameter $\beta$, with the limiting that it does not reach values that allow it to leave the region determined by $\sigma$. In summary, in each region, we can vary one or both $\sigma$ and $\beta$ parameters at the same time, and if this makes the electric field of the model increase, for example, then the efficiency value also increases.

It would be interesting to study these black holes in the context of stability due to perturbations and see how it is related to phase transitions and the $\sigma$ value. 


\section{Acknowledgments}

L.B. was supported by DIUFRO through the project DI19-0052.

\section{References}

[1] S. I. Kruglov, Notes on Born-Infeld-type electrodynamics, Mod.Phys. Lett. A 32 no.36, 1750201 (2017).

[2] M. Born and L. Infeld, Foundations of the new field theory, Proc. Roy. Soc. Lond. A 144, no. 852, 425 (1934).

[3] B. Hoffmann, Gravitational and electromagnetic mass in the Born-Infeld electrodynamics, Phys. Rev. 47, no. 11, 877 (1935).

[4] I. H. Salazar, A. Garcia and J. Plebanski, Duality Rotations and Type D Solutions to Einstein Equations With Nonlinear Electromagnetic Sources, J. Math. Phys. 28, 2171 (1987).

[5] M. Cataldo and A. Garcia, Three dimensional black hole coupled to the BornInfeld electrodynamics, Phys. Lett. B 456, 28 (1999).

[6] R. Garcia-Salcedo and N. Breton, Born-Infeld cosmologies, Int. J. Mod. Phys. A 15, 4341 (2000).

[7] S. Fernando and D. Krug, Charged black hole solutions in Einstein-Born-Infeld gravity with a cosmological constant, Gen. Rel. Grav. 35, 129 (2003).

[8] O. Miskovic and R. Olea, Thermodynamics of Einstein-Born-Infeld black holes with negative cosmological constant, Phys. Rev. D 77, 124048 (2008).

[9] A. A. Tseytlin, On nonAbelian generalization of Born-Infeld action in string theory, Nucl. Phys. B 501, 41 (1997).

[10] S. Cecotti and S. Ferrara, Supersymmetric Born-infeld Lagrangians, Phys. Lett. B 187, 335 (1987).

[11] C. G. Callan and J. M. Maldacena, Brane death and dynamics from the BornInfeld action, Nucl. Phys. B 513, 198 (1998).

[12] H. H. Soleng, Charged black points in general relativity coupled to the logarithmic U(1) gauge theory, Phys. Rev. D 52, 6178 (1995).

[13] S. H. Hendi, Asymptotic charged BTZ black hole solutions, JHEP 1203, 065 (2012). 
[14] P. Gaete and J. Helayël-Neto, Remarks on nonlinear Electrodynamics, Eur. Phys. J. C 74, no.11, 3182 (2014).

[15] S. I. Kruglov, On Generalized Logarithmic Electrodynamics, Eur. Phys. J. C 75, no. 2,88 (2015).

[16] S. I. Kruglov, Modified nonlinear model of arcsin-electrodynamics, Commun. Theor. Phys. 66, no. 1, 59 (2016).

[17] S. I. Kruglov, Nonlinear arcsin-electrodynamics and asymptotic Reissner-Nordström black holes, Annalen Phys. 528, (2016) 588. doi:10.1002/andp.201600027

[18] S. I. Kruglov, On a model of magnetically charged black hole with nonlinear electrodynamics, Universe 4, no. 5, (2018) 66. doi:10.3390/universe4050066

[19] E. Ayon-Beato and A. Garcia, Regular black hole in general relativity coupled to nonlinear electrodynamics, Phys. Rev. Lett. 80, 5056 (1998).

[20] E. Ayon-Beato and A. Garcia, New regular black hole solution from nonlinear electrodynamics, Phys. Lett. B 464, 25 (1999).

[21] K. A. Bronnikov, Regular magnetic black holes and monopoles from nonlinear electrodynamics, Phys. Rev. D 63, 044005 (2001).

[22] I. Dymnikova, Regular electrically charged structures in nonlinear electrodynamics coupled to general relativity, Class. Quant. Grav. 21, 4417 (2004).

[23] L. Balart and E. C. Vagenas, Regular black hole metrics and the weak energy condition, Phys. Lett. B 730, 14 (2014).

[24] L. Balart and E. C. Vagenas, Regular black holes with a nonlinear electrodynamics source, Phys. Rev. D 90, no. 12, 124045 (2014).

[25] L. Balart and S. Fernando, A Smarr formula for charged black holes in nonlinear electrodynamics, Mod. Phys. Lett. A 32, no. 39, 1750219 (2017).

[26] I. Gullu and S. H. Mazharimousavi, Black Holes in Double-Logarithmic Nonlinear Electrodynamics, arXiv:2010.04603 [gr-qc]].

[27] S. I. Kruglov, Born-Infeld-type electrodynamics and magnetic black holes, Annals of Phys. 383, 550 (2017).

[28] S. Gunasekaran, R. B. Mann and D. Kubiznak, Extended phase space thermodynamics for charged and rotating black holes and Born-Infeld vacuum polarization, JHEP 1211, 110 (2012). 
[29] R. Ruffini, Y. B. Wu and S. S. Xue, Einstein-Euler-Heisenberg Theory and charged black holes,' Phys. Rev. D 88, 085004 (2013).

[30] S. W. Hawking and G. F. R. Ellis, The Large Scale Structure of Space-Time. doi:10.1017/CBO9780511524646

[31] D. Kubiznak and R. B. Mann, P-V criticality of charged AdS black holes, JHEP 1207, 033 (2012).

[32] A. Rajagopal, D. Kubizňák and R. B. Mann, Van der Waals black hole, Phys. Lett. B 737, 277 (2014).

[33] R. A. Hennigar and R. B. Mann, Reentrant phase transitions and van der Waals behaviour for hairy black holes, Entropy 17, no. 12, 8056 (2015).

[34] J. Xu, L. M. Cao and Y. P. Hu, P-V criticality in the extended phase space of black holes in massive gravity, Phys. Rev. D 91, no. 12, 124033 (2015).

[35] J. X. Mo and W. B. Liu, P-V criticality of topological black holes in LovelockBorn-Infeld gravity, Eur. Phys. J. C 74, no. 4, 2836 (2014).

[36] S. H. Hendi, S. Panahiyan and B. Eslam Panah, $P-V$ criticality and geometrical thermodynamics of black holes with Born-Infeld type nonlinear electrodynamics, Int. J. Mod. Phys. D 25, no. 01, 1650010 (2015).

[37] J. X. Mo, G. Q. Li and X. B. Xu, Effects of power-law Maxwell field on the critical phenomena of higher dimensional dilaton black holes, Phys. Rev. D 93, no. 8, 084041 (2016).

[38] M. Zhang \& W Liu, Coexistent physics of massive black holes in the phase transitions, arXiv: 1610.03648

[39] G. Q. Li, Effects of dark energy on P-V criticality of charged AdS black holes, Phys. Lett. B 735, 256 (2014).

[40] R. G. Cai, L. M. Cao, L. Li and R. Q. Yang, P-V criticality in the extended phase space of Gauss-Bonnet black holes in AdS space, JHEP 1309, 005 (2013).

[41] M. Azreg-Aïnou, Black hole thermodynamics: No inconsistency via the inclusion of the missing $P-V$ terms, Phys. Rev. D 91, 064049 (2015).

[42] J. Sadeghi, B. Pourhassan and M. Rostami, P-V criticality of logarithm-corrected dyonic charged AdS black holes, Phys. Rev. D 94, no. 6, 064006 (2016).

[43] D. Kubiznak, R. B. Mann and M. Teo, Black hole chemistry: thermodynamics with Lambda, Class. Quant. Grav. 34, no. 6, 063001 (2017). 
[44] C. V. Johnson, Holographic Heat Engines, Class. Quant. Grav. 31, 205002 (2014).

[45] C. V. Johnson, Born-Infeld AdS black holes as heat engines, Class. Quant. Grav. 33, no. 13, 135001 (2016).

[46] C. V. Johnson, Taub-Bolt heat engines, Class. Quant. Grav. 35, no. 4, 045001 (2018).

[47] C. V. Johnson, An exact efficiency formula for holographic heat engines, Entropy 18, 120 (2016).

[48] B. Chandrasekhar and P. K. Yerra, Heat engines for dilatonic Born-Infeld black holes, Eur. Phys. J. C 77, no. 8, 534 (2017) .

[49] H. Xu, Y. Sun and L. Zhao, Black hole thermodynamics and heat engines in conformal gravity, Int. J. Mod. Phys. D 26, no. 13, 1750151 (2017).

[50] J. X. Mo, F. Liang and G. Q. Li, Heat engine in the three-dimensional spacetime, JHEP 1703, 010 (2017).

[51] H. Liu and X. H. Meng, Effects of dark energy on the efficiency of charged AdS black holes as heat engines, Eur. Phys. J. C 77, no. 8, 556 (2017).

[52] S. H. Hendi, B. Eslam Panah, S. Panahiyan, H. Liu and X.-H. Meng, Black holes in massive gravity as heat engines, Phys. Lett. B 781, 40 (2018).

[53] R. A. Hennigar, F. McCarthy, A. Ballon and R. B. Mann, Holographic heat engines: general considerations and rotating black holes, Class. Quant. Grav. 34, no. 17, 175005 (2017).

[54] L. Balart and S. Fernando, Non-linear black holes in $2+1$ dimensions as heat engines, Phys. Lett. B 795, 638 (2019).

[55] P. Niau Akmansoy and L. G. Medeiros, Constraining Born-Infeld-like nonlinear electrodynamics using hydrogen's ionization energy, Eur. Phys. J. C 78, no.2, 143 (2018).

[56] P. Niau Akmansoy and L. G. Medeiros, Constraining nonlinear corrections to Maxwell electrodynamics using $\gamma \gamma$ scattering, Phys. Rev. D 99, no.11, 115005 (2019).

[57] M. J. Neves, J. B. de Oliveira, L. P. R. Ospedal and J. A. Helayël-Neto, Dispersion Relations in Non-Linear Electrodynamics and the Kinematics of the Compton Effect in a Magnetic Background, [arXiv:2101.03642 [hep-th]].

[58] W. Yi-Huan, Energy and first law of thermodynamics for Born-Infeld-anti-deSitter black hole, Chin. Phys. B 19, 090404 (2010). 
[59] D. Kastor, S. Ray and J. Traschen, Enthalpy and the Mechanics of AdS Black Holes, Class. Quant. Grav. 26, 195011 (2009).

[60] L. Smarr, Mass formula for Kerr black holes, Phys. Rev. Lett. 30, 71 (1973). Erratum: [Phys. Rev. Lett. 30, 521 (1973)].

[61] C. H. Nam, Thermodynamics and phase transitions of non-linear charged black hole in AdS spacetime, Eur. Phys. J. C 78, no.7, 581 (2018).

[62] X. M. Kuang, B. Liu and A. Övgün, Nonlinear electrodynamics AdS black hole and related phenomena in the extended thermodynamics, Eur. Phys. J. C 78, no.10, 840 (2018).

[63] A. Jawad and A. Khawer, Thermodynamic consequences of well-known regular black holes under modified first law, Eur. Phys. J. C 78, no.10, 837 (2018).

[64] D. V. Singh and S. Siwach, On Thermodynamics and Statistical Entropy of Bardeen Black Hole,

[65] M. S. Ma and R. Zhao, Corrected form of the first law of thermodynamics for regular black holes, Class. Quant. Grav. 31, 245014 (2014).

[66] C. Lan, Y. G. Miao and H. Yang, Quasinormal Modes and Thermodynamics of Regular Black Holes, arXiv:2008.04609 [gr-qc]]. 